Macedonian Pharmaceutical Bulletin, 66 (Suppl 1) 97 - 98 (2020)

Online ISSN 1857 - 8969

UDC: 615.281:546.26-162

DOI: 10.33320/maced.pharm.bull.2020.66.03.048

Short communication

\title{
Pharmaceutical uses of graphene
}

\author{
Despina Spasevska Kovacki
}

\section{Alkaloid AD Skopje, Blvd. Aleksandar Makedonski 12, 1000 Skopje, Republic of North Macedonia}

\section{Introduction}

Graphene is a material composed of pure carbon, similar to graphite but with characteristics that make it extraordinarily light and strong. A sheet of one square meter of graphene weighs $0.77 \mathrm{mg}$. Its strength is 200 times greater than that of steel and its density is similar to that of carbon fiber. All these make it resist high bending forces without breaking. It is one of the most conductive materials for electricity and heat, which makes it the perfect material for many industries.

Graphene oxide (GO), the oxidized form of graphene, holds great potential as a component of biomedical devices, deriving utility from its ability to support a broad range of chemical functionalities and its exceptional mechanical, electronic and thermal properties.

Some of the uses of graphene in pharmacy are listed in the following text.

Wang at al. (2019) have shown that graphenebased nanomaterials (GNMs) are successfully used for the removal of prominent antibiotics, which absorb onto GNMs mainly through $\pi-\pi$ interaction, electrostatic interaction, hydrophobic interaction and hydrogen bonding.

Graphene is a magnificent bactericidal material as it avoids the generation of microorganisms, such as bacteria, viruses, and fungi, by damaging their cell membranes between its outer layers. When compared to different derivatives of Graphene, Graphene Oxide and reduced Graphene Oxide shows the best antibacterial effects. GO can also be used as a compound with silver nanoparticles to increase antibacterial properties even further (Song et al., 2016).

Functionalized graphene can be used to carry chemotherapy drugs to tumors for cancer patients. Graphene based carriers target cancer cells better and reduced and decreased toxicity of the effected healthy cells.

Patel at al. (2016) have shown that hydrophobic cancer drug delivery is facilitated by hydrophobic interactions between the drug and graphene oxide molecules, leading to water dispersible graphene loaded with cancer drugs with otherwise limited water solubility. It is also an excellent photothermal agent for photothermal therapy. Its physiochemical properties can be harnessed to facilitate photothermal and photodynamic therapy to treat superficial or deep tissue cancers.

In vitro investigations and in vivo proof of principle small animal studies provide evidence that graphene-based formulations could serve as versatile delivery agents. The results suggest that morphology of graphene as well as the functional groups employed to improve its water dispersibility may play an important role toward the development of an optimum formulations for efficacious anticancer therapies.

Drug delivery is not limited to cancer treatment. Anti-inflammatory drugs have also been carried by graphene and chitosan combinations and yielded promising results (Unnithan et al., 2017).

\footnotetext{
*dkovacki@alkaloid.com.mk
} 
Gene delivery is a method used to cure some genetic diseases by bringing foreign DNA into cells. Graphene oxide (GO) modified by polyethyleneimine can be used for these purposes is expected to show low cytotoxicity, as it did in the drug delivery case (Bao et al., 2011).

Holt et al. (2016) have shown that GO or a GOcontaining composite can be used to coat traditional osteogenic materials/scaffolds. GO can be noncovalently or covalently loaded for controlled release of osteogenic molecules. GO can be incorporated with other materials to form composites with enhanced properties for osteogenesis.

Graphene oxide/collagen (GO-COL) aerogel exhibit relatively good biocompatibility and osteogenic ability in vitro and in vivo, which make it a promising bioscaffold for bone tissue engineering and regenerative medicine (Liu et al., 2019).

A group of researchers (Kidambi et al., 2017) showed that graphene (in a form of graphene membrane) can be used to filter the blood from wastes, drugs and chemicals as well. Graphene's superiority in this case is that it is 20 times thinner than traditional membranes which leads to significant decrease in the time spent in the dialysis for the patients.

An injectable hydrogel scaffold made of polyethylene glycol-melamine-hyaluronic acid-GO (PEG-MEL/HA-SH/GO) was found to have excellent conductivity and anti-fatigue properties for cardiac tissue engineering. When injected into the infarcted area of the myocardium, adipose-tissuederived stromal cells encapsulated within these PEG-MEL/HA-SH/GO hydrogels improved the functional performance of the heart (Lakshmanan and Maulik, 2018).

\section{Conclusion}

The application of graphene is virtually unlimited and promises to revolutionize many fields: from electronics and computing to construction and health.
Graphene is called the "wonder material" for a reason. There are tens of researches about it which are not published yet, but may change the world tomorrow.

\section{References}

Bao, H., Pan, Y., Ping, Y., Sahoo, N.G., Wu, T., Li, L., Li, J., Gan, L.H., 2011. Chitosan-functionalized graphene oxide as a nanocarrier for drug and gene delivery. Wiley 7, 1569-1578.

Holt, B.D., Wright, Z.M., Arnold, A.M., Sydlik, S.A., 2016. Graphene oxide as a scaffold for bone regeneration. WIREs Nanomed Nanobiotechnol. 9, e1437.

Kidambi, P., Jang, D., Idrobo, J.C., Boutilier, M.S.H., Wang, L., Kong, J., Karnik, R., 2017. Nanoporous atomically thin graphene membranes for desalting and dialysis applications. Adv. Materials 29.

Lakshmanan, R., Maulik, N., 2018. Graphene-based drug delivery systems in tissue engineering and nanomedicine. Canadian Journal of Physiology and Pharmacology 96, 869-878

Liu, S., Zhou, C., Mou, S., Li, J., Zhou, M., Zeng, Y., Luo, C., Sun, J., Wang, Z., Xu, W., 2019. Biocompatible graphene oxide-collagen composite aerogel for enhanced stiffness and in situ bone regeneration. Sci. Direct C105.

Patel, S., Lee, S., Lalwani, G., Suhrland, C., Chowdhury, S.M., Sitharaman, B., 2016. Graphene-based platforms for cancer therapeutics. Future Sci. 7, 101116.

Song, B., Zhang, C., Zeng, G., Gong, J., Chang, Y., Jiang, Y., 2016. Antibacterial properties and mechanism of graphene oxide-silver nanocomposites as bactericidal agents for water disinfection. Sci. Direct. 604, 167176.

Unnithan, A.R., Ramachandra, A., Sasikala, K., Park, C.H., Kim, C.S., 2017. A unique scaffold for bone tissue engineering: An osteogenic combination of graphene oxide-hyaluronic acid-chitosan with simvastatin. Sci. Direct 46, 182-191.

Wang, X., Yin, R., Zeng, L., Zhu, M., 2019. A review of graphene-based nanomaterials for removal of antibiotics from aqueous environments. Sci. Direct 253, 100-110. 